Konditionierte Verstärker, Nicht-Sättigung und das Lernen zu Verbrauchen

\section{Neues aus der Konsumtheorie}

\author{
Die Erklärungsbereiche von Ökonomie und Psychologie überschneiden sich \\ wahrscheinlich nirgends so wie beim Konsumverhalten. Die Ökonomik täte gut \\ daran, in einen intensiveren Diskurs mit Nachbardisziplinen zu treten (1), statt \\ sich auf die Konsumentensouveränität zurückzuziehen. Denn auch menschliche \\ Bedürfnisse sind Wandlungs- und Lernprozessen unterworfen. Dies ist gerade \\ angesichts der bestehenden Umweltprobleme des Konsums von Bedeutung.
}

$\mathrm{D}$

Von Ulrich Witt

ie Ökonomik gibt sich gegenüber dem Konsum und seiner Schlüsselrolle für Sinn und Zweck des modernen Wirtschaftsgeschehens recht naiv. Zum einen interpretiert sie einen steigenden Konsum als undiskutierten Endzweck. Zum anderen unterstellt sie, dass ein bei steigendem Einkommen wachsendes Konsumpotenzial stets durch eine wachsende Nachfrage ausgeschöpft wird. Zur Begründung wird selten mehr als die These der „Unersättlichkeit menschlicher Bedürfnisse“ angeführt. Aber was besagt diese These schon? Aus empirischen Untersuchungen über den Konsum und die Einkommenselastizität von Konsumgütern ist bekannt, dass es erhebliche Veränderungen in der Zusammensetzung der nachgefragten Güter gibt, wenn das Einkommen steigt (2). Inferiore Güter werden relativ oder sogar absolut weniger, superiore Güter dagegen mehr nachgefragt. Aber diese Beschreibung bzw. Klassifizierung erklärt nicht, warum die Einkommenselastizitäten so sind, wie sie sind.

Die empirisch geschätzten Elastizitäten reflektieren außerdem auch Effekte von Konsuminnovationen. Mit der ständigen Vermehrung der Vielfalt an Gütern und Dienstleistungen geht ein qualitativer Wandel der Nachfrage einher. Wie kommt es, dass Nachfrager stets weitere, neue Angebote begehren? Ohne materielle Hypothesen darüber, was Menschen konsumieren und warum, kommt man hier nicht weiter. Die ökonomische Nutzenbzw. Präferenztheorie lässt offen, wofür Menschen Präferenzen haben. Will man diesbezügliche Hypothesen nicht neu erfinden, muss man sie, wie nachfolgend skizziert, aus dem Dialog mit Psychologie, Sozialpsychologie und - was die genetischen Einflüsse angeht - der Soziobiologie gewinnen (3). Wie sich zeigen wird, hat eine so fundierte Konsumtheorie erhebliche Substanz, nicht zuletzt im Hinblick auf die moral-philosophischen Fragen, die ein (unersättlich?) wachsender Konsum aufwirft. Solche Fragen haben ursprünglich im viel geschmähten utilitaristischen Forschungsprogramm eine wichtige Rolle gespieIt (4). In Anbetracht der vielfältigen Externalitätenprobleme des wachsenden Konsums scheint eine Wiederbelebung dieser Debatte durchaus angeraten.

\section{- Was wird konsumiert? Die Theorie}

Um Anknüpfungspunkte für einen Dialog mit den Verhaltenswissenschaften zu erhalten, muss man das abstrakte Niveau der Präferenztheorie verlassen. Konsum war ursprünglich gleichbedeutend mit Verbrauchen im Sinne eines Aktes des Aufzehrens, sagen wir eines Nahrungsmittels. Heute fällt darunter aber zum Beispiel auch der Konsum von solchen langlebigen Gütern wie einem Fernsehapparat. Von Verzehr könnte man da allenfalls hinsichtlich der Dienstleistungen sprechen, die solche Güter erbringen. Tatsächlich ist ein Fernseher, dessen Bildschirm dunkel bleibt und der daher keine Dienstleistung abgibt, normalerweise nicht ein besonders begehrter Gegenstand. Die enormen phänomenologischen Unterschiede zu betonen, die sich hinter dem abstrakten Begriff Konsum verbergen, mag im Lichte der Abstraktionsstrategie der modernen Präferenztheorie befremdlich erscheinen. Eine solche Orientierung hat jedoch viel mit dem Erklärungsprogramm des sensorischen Utilitarismus eines Jeremy Bentham gemeinsam, das am Anfang der utilitaristischen Ökonomik stand.

Konsum im Sinne direkten Verzehrs ist ein anschaulicher Fall. Was direkt verzehrt wird, ist eine relativ kleine Zahl von Gütern. Sie dienen einigen wenigen Elementarbedürfnissen nach Luft zum Atmen, Nahrung zum Essen und wäss- rigen Lösungen zum Trinken. Die Befriedigung dieser genetisch angelegten Bedürfnisse ist eine physiologische Notwendigkeit für die Aufrechterhaltung der Lebensfunktionen des Organismus. Es liegt nahe, nach weiteren genetischen Einflüssen auf das Konsumverhalten zu suchen. Tatsächlich lassen sich einige weitere Elementarbedürfnisse identifizieren, nämlich jene nach Aufrechterhaltung der Körpertemperatur, nach Schlaf, physischer Aktivität, mentaler Stimulation, sexueller Befriedigung, Zuneigung und Fürsorge und schließlich nach sozialer Anerkennung oder Status. Das Streben nach Befriedigung dieser Bedürfnisse findet sich ebenfalls in allen menschlichen Kulturen ebenso wie bei den höher entwickelten Arten im Tierreich. Im Unterschied zur erstgenannten Gruppe werden die Elementarbedürfnisse der zweiten Gruppe jedoch nicht durch direkten Verzehr von Gütern befriedigt.

\section{Ein Beispiel}

Nehmen wir das Elementarbedürfnis Aufrechterhaltung der Körpertemperatur. Wenn die Außentemperatur stark von der Körpertemperatur abweicht, muss das Bedürfnis durch Mittel zum Ausgleich des Temperaturunterschieds befriedigt werden. Im Tierreich geschieht dies weitgehend endosomatisch, beim Menschen auf exosomatischem Wege. Dieser Unterschied ist Ausdruck menschlicher Intelligenz, die die Herstellung und den Gebrauch von Instrumenten und Werkzeugen ermöglicht. Zufällige Entdeckung, Phantasie, Versuch und Irrtum haben eine große Zahl von Artefakten hervorgebracht, die wir gebrauchen - oder genauer, deren Dienstleistungen wir gebrauchen - um die genannten Elementarbedürfnisse (besser) zu befriedigen. Auch für die Befriedigung des Bedürfnisses nach sozialer Anerkennung und Status sind reichlich Mittel erfunden worden.

Diese Bedürfnisse stehen in Verbindung mit dem, was in der behavioristischen Psychologie Primärverstärker genannt wird (5). In Experimenten kann man arten-übergreifend beobachten, dass Handlungen, die zu einer Befriedigung dieser Bedürfnisse führen oder beitragen, in der Folge mit verstärkter Häufigkeit auftreten. Voraussetzung ist, dass eine Befriedigung durch eine solche Handlung überhaupt möglich ist. Dies ist nicht der Fall, wenn der Organismus hinsichtlich dieses Bedürfnisses bereits gesättigt ist. Sättigung ist also eine wichtige Verhaltenskontingenz. Bei Konsumgütern, die zur Befriedigung eines Bedürfnisses direkt verzehrt werden, ist sie evident. Aber auch 
hinsichtlich der zweiten Gruppe von Elementarbedürfnissen ist Sättigung ein relativ klares Phänomen. Es bezieht sich dort jedoch auf die Wirkung der Dienstleistungen, die die Instrumente abgeben können.

Im Beispiel des Bedürfnisses nach Aufrechterhaltung der Körpertemperatur tritt eine „Sättigung“ ein, wenn durch geeignete Mittel der Temperaturunterschied zur Außentemperatur ausgeglichen ist. Der „Konsum“ (im Sinne von Erwerb) der Instrumente selbst ist nicht direkt sättigbar. Wie viele Instrumente zur Befriedigung ein und desselben Elementarbedürfnisses man erwirbt, hängt deshalb von anderen, vermutlich kognitiven Faktoren ab. Hinter diesen kognitiven Faktoren verbirgt sich die individuelle Wahrnehmung einer möglicherweise größeren Zahl von weiteren ZielMittel-Zusammenhängen, für die ein Konsumgut in den Augen eines Konsumenten instrumentalisiert werden kann. Ein Wintermantel befriedigt wohl nicht nur das Bedürfnis nach Aufrechterhaltung der Körpertemperatur, sondern kann vielleicht auch zur mentalen Stimulation (ästhetischer Genuss) und zur Geltendmachung eines sozialen Status (soziale Anerkennung) beitragen. Im Falle eines solchen „Kombi“-Gutes werden dann gleich mehrere und wahrscheinlich unterschiedliche Sättigungsgrenzen relevant: Während ein bestimmter Konsumumfang das eine Bedürfnis vielleicht schon sättigt, sind andere Bedürfnisse damit noch nicht notwendig gesättigt, oder sind möglicherweise überhaupt nicht zu sättigen. Die Folge: Wenn es das Einkommen zulässt, hängen mehrere Mäntel im Schrank, wo es zur Aufrechterhaltung der Körpertemperatur allein nur eines einzigen bedürfte.

\section{Bedürfnisse und ihr Wandel}

Die elementaren Bedürfnisse sind Teil des menschlichen Erbes. Von der üblichen genetischen Varianz abgesehen sind sie interindividuell weitgehend gleichartig. Individuelles Begehren und Konsumieren nimmt jedoch sehr unterschiedliche Formen an. Was führt von den interindividuell relativ gleichartigen Elementarbedürfnissen zu den individuellen Formen des Konsumierens? Zur Erklärung kann man auf Lernhypothesen zurückgreifen. Der Lernmechanismus, der für die Entstehung und Veränderung von nicht ererbten Bedürfnissen verantwortlich ist, ist ebenfalls genetisch angelegt. In den Verhaltenswissenschaften wird er „Konditionierungslernen“ genannt.
Wird die oben bereits erwähnte Erfahrung einer primären Verstärkung, zum Beispiel die Befriedigung von Hunger durch eine Nahrungsaufnahme immer wieder mit denselben, an sich neutralen Reizen kombiniert, dann wird eine Assoziation zwischen der primären Verstärkung und den an sich neutralen Reizen gelernt. Letztere werden dadurch selbst zu so genannten konditionierten oder sekundären Verstärkern. Das heißt, die relative Häufigkeit eines Verhaltens, auf das die konditionierte Verstärkung folgt, nimmt dann auch ohne Anwesenheit der ursprünglichen, primären Verstärkung zu. Die sekundären Verstärker wirken allerdings nur temporär und verlieren ihre eigenständig verstärkende Wirkung wieder, wenn sie nicht gelegentlich wieder in Verbindung mit der ursprünglichen Verstärkung auftreten.

Der größte Teil des Erlernens von erworbenen Bedürfnissen findet während der Sozialisationsphase statt. Welche Assoziationen wir lernen und welche Bedürfnisse wir dabei entwickeln, wird stark von den Gegebenheiten unserer sozialen und kulturellen Umgebung beeinflusst. Die Komplexität der individuellen Lerngeschichte erklärt die außerordentliche inter-individuelle Varianz der Bedürfnisse zu jedem Zeitpunkt ebenso, wie die individuelle Veränderlichkeit über die Zeit hinweg.

Ererbte und erlernte Bedürfnisse folgen daher einem unterschiedlichen Sättigungsmuster. Kulturell erworbene Bedürfnisse unterliegen keinem Wechselspiel von Deprivation und Sättigung. Gesättigt wird allenfalls der primäre Verstärker, zu dem die Assoziation gelernt wurde. Auch hier muss daher die Frage, wie viel von diesen Gütern konsumiert wird, von anderen, vermutlich kognitiven Faktoren abhängig gemacht werden. Infolge dessen machen sich auf dem Weg von einem ungesättigten Bedürfnis zu einem konkreten Kon-

\section{Einen Überblick über alle bisher erschienenen Hefte des Informationsdienstes}

\section{"Ökologisches Wirtschaften"}

\section{finden Sie auf unseren Internetseiten unter der Adresse: \\ http://www.oekom.de}

Wir freuen uns auf Ihren Besuch! sumakt noch Faktoren geltend, die durch die skizzierte Theorie der Bedürfnisse allein nicht erklärt werden.

Im Falle der Instrumente, die zur Bedürfnisbefriedigung eingesetzt werden, ist die Auswahl von zweierlei abhängig: einerseits von den Zielerreichungstechniken, die dem Individuum bekannt sind; andererseits von der Verfügbarkeit der Mittel, so wie sie subjektiv wahrgenommen werden. Auf dieser kognitiven Ebene kommen also die altbekannten Konzepte von Produktionsmöglichkeiten und -beschränkungen zu ihrer Geltung.

\section{- Einige Implikate: Nahrungsmittelkonsum...}

Die Befriedigung der erworbenen Bedürfnisse gewinnt umso mehr Einfluss auf das Konsumverhalten, je mehr die Elementarbedürfnisse durch relative Sättigung zurücktreten. Güter, die der Befriedigung erworbener Bedürfnisse dienen können, sind daher - scheinbar paradox - in der Gruppe der superioren Güter zu finden: Mit steigendem Realeinkommen, das eine zunehmende relative Sättigung der Elementarbedürfnisse ermöglicht, machen vermehrt die kulturell erworbenen Bedürfnisse, die selbst nicht sättigbar sind, ihren Einfluss auf den Konsum geltend. Damit sind wir beim Thema Wirtschaftswachstum und Konsum. Steigende Einkommen bedeuten wegen des Sättigungsproblems nicht notwendig steigenden Konsum. Die entscheidende Frage ist, aus welchen Gründen und wo eine Sättigung der Nachfrage eintritt und wo nicht.

Im einfachsten Fall - im Zusammenhang mit der Befriedigung von Elementarbedürfnissen - sind physiologische Deprivations- und Sättigungsmuster wirksam. Wie dargelegt, betrifft dies eigentlich nur den Nahrungsmittelkonsum. Hier gibt es eine klare Sättigungsgrenze, die viele Menschen auf dieser Erde noch nicht erreicht haben. In den hochentwickelten Ländern ist beim durchschnittlichen Einkommen die Grenze längst erreicht. Aber selbst in diesen Ländern wächst der Nahrungsmittelkonsum in realen Termen noch weiter. Wie ist das möglich? Natürlich kann man das Sättigungsgefühl ignorieren und weiter essen. Oder man kann mehr Nahrungsmittel konsumieren (käuflich erwerben), ohne sie jedoch (vollständig) zu verzehren - manche nennen das Verschwendung. Mit zunehmendem Pro-Kopf-Einkommen dürfte dieser Fall für das weitere Wachstum des Nahrungsmittelkonsums durchaus eine 
Rolle spielen. Eine wichtige Ursache ist, dass Nahrung oft einen Teil der schon erwähnten „Kombi“Güter darstellt.

Eine signifikante Rolle für das ungebrochene Wachstum des Nahrungsmittelkonsums spielen auch technologische Entwicklungen, die auf die Vermeidung von Sättigungswirkungen zielen. Den Paradefall stellt die Geschichte des Zuckers und der Süßstoffe dar (6). Mittels des Übergangs von Zucker auf Süßstoffe wird die Sättigung gewissermaßen durch Manipulation der Nahrung hinausgeschoben und dadurch weiteres Konsumwachstum möglich.

\section{- ... positionale Güter ...}

Erst recht ergibt sich Konsumwachstum dann, wenn Sättigung nicht, oder nur schwer möglich ist. Dies ist zunächst, wie oben erwähnt, bei einigen der Elementarbedürfnisse der Fall. Nehmen wir das Bedürfnis nach sozialer Anerkennung und Status. Streben nach möglichst hohem Status und Statussignalisierung durch demonstrativen Konsum sind eine instabile Kombination: Einkommenssteigerungen lösen eine Aufholkonkurrenz im Konsum aus, wie schon Hirsch in seiner Theorie der positional goods erkannte (7). Die Folge ist eine Spirale wie beim Rüstungswettlauf, die zu einem immer aufwändigeren Konsum führt. In der Regel zielt er auf „Kombi“-Güter ab, die eine oder mehrere Komponenten besitzen, mit denen Status signalisiert werden kann. Der Konsum kann hier immer weiter steigen, auch wenn die Nachfrage nach allen anderen Komponenten im Prinzip schon längst gesättigt ist.

Ein weiteres, schwer sättigbares Elementarbedürfnis, das deutliche Spuren in der modernen Konsumwelt hinterlässt, ist das Bedürfnis nach mentaler Stimulation: Unterhaltung, Erlebnis-Konsum, Freizeitindustrie, Medien und vor allem Mobilität, soweit diese konsumtiven Charakter hat, erlangen in der Wertschöpfung von Volkswirtschaften mit hohem Pro-Kopf-Einkommen immer größere Bedeutung (8). Auch bezüglich der Befriedigung dieser Bedürfnisse ergibt sich eine latente Instabilität. Dem relativen Charakter der mentalen Stimulation entsprechend müssen immer neue und immer stärkere Stimuli die Abnutzung durch Gewöhnung kompensieren.

\section{-... und instrumentelle Güter}

Bei Gütern mit Instrumentcharakter tritt - wie oben erwähnt - keine direkte Sättigung auf. Es kommt nur darauf an, dass sie vom Konsumenten auf der kognitiven Ebene als tauglich eingestuft werden, ein noch ungesättigtes Bedürfnis zu befriedigen. Dann steht dem Auf- und Ausbau von Beständen solcher Konsumgüter nichts im Wege entsprechende Einkommenszuwächse immer vorausgesetzt. So machen denn solche Güter mit instrumentellem Charakter auch den Löwenanteil unter den neuen Gütern aus, wie die Welle der consumer electronics der letzten Jahrzehnte eindrücklich veranschaulicht. Das Problem der Hersteller ist, die Konsumenten immer wieder von der Nützlichkeit der neuen Güter als Instrumente für die Befriedigung existierender oder vielleicht erst noch zu erlernender Bedürfnisse zu überzeugen. Da bei der ungeheuren Vielzahl von Gütern mit instrumentellem Charakter inzwischen das zu ihrer Beurteilung erforderliche Konsumwissen schon längst über das Maß hinausgeht, das jeder einzelne noch verarbeiten kann, ist die Folge, dass die Konsumenten sich zunehmend in ihrem Konsumentenwissen spezialisieren. Da Konditionierungslernen wie auch kognitives Lernen den Geschmack, das Wissen und letztlich die Detailliertheit der Bedürfnisse nur für solche Dinge verfeinern, die unsere Aufmerksamkeit finden, bedeutet Spezialisierung im Konsum weit mehr als nur eine Teilung des Wissens und der Fähigkeiten wie bei der Spezialisierung in der Produktion. Es bedeutet auch eine zunehmende Ausdifferenzierung unserer erlernten Bedürfnisse, intrawie inter-individuell.

\section{Moral-philosophische Legitimati- onsprobleme}

Beim gut-situierten Teil der Menschen auf dieser Erde wird durch technologische Entwicklungen die Sättigung von Elementarbedürfnissen hinausgezögert. Im Fall der „Kombi“-Güter wird der Konsum um einiger ungesättigter Komponenten willen noch ausgedehnt. Im Fall der Güter mit Instrumentcharakter wird der Besitzerwerb auch dort fortgeführt, wo die Dienstleistungen jedes weiteren Gutes nur noch bruchteilhaft in Anspruch genommen werden. All dies geschieht vor dem Hintergrund einer doppelten Problematik. Zum einen erlaubt die globale Einkommensverteilung einem beträchtlichen Teil der Weltbevölkerung kaum, wenigstens genügend Nahrungsmittel für das nackte Überleben konsumieren zu können. Zum anderen verursacht der global wachsende Konsum massive externe Effekte in der Umwelt, eine Hypothek für zukünftige Generationen. Ist dies zu rechtfertigen in Anbetracht der Tatsache, dass die reichsten Völker vorleben, wie wenig sie die hinausgeschobene Sättigung der
Elementarbedürfnisse, der Erwerb kulturell bedingter, erlernter Bedürfnisse und die Spezialisierung im Konsum glücklicher zu machen scheinen (9)?

Um keine Missverständnisse zu provozieren: Das Anliegen ist hier nicht, der Reglementierung der Konsumenten das Wort zu reden. Vielmehr geht es darum, über das Zustandekommen und die Voraussetzungen von Konsumwünschen wissenschaftlich zu diskutieren. Das Schlagwort von der Konsumentensouveränität darf nicht zur Devise werden, die Bedürfnisse und Handlungsmotive tabuisiert. Eine Menge von dem, was wir heute konsumieren, haben wir erst durch Konditionierungslernen und kognitives Lernen zu schätzen begonnen. Würde man uns die Möglichkeit nehmen, diese Bedürfnisse zu befriedigen, würden wir dies sicherlich als (Nutzen-) Einbuße erleben. Aber hätten wir nicht gelernt, was wir als Konsumenten lernen, würde uns die Einbuße nichts bedeuten. Vielleicht könnte diese Einsicht die Bereitschaft fördern, das moralische Legitimationsproblem ernst zu nehmen.

\section{Anmerkungen}

(1) Ansätze dazu gab es in den frühen Arbeiten von Katona. Vgl. Katona, G.: The Powerful Consumer. New York 1960. (2) Aversi, R./ Dosi, G./ Fagiolo, G./ Meacci, M./ Olivetti, C.: Demand Dynamics with Socially Evolving Preferences. In: Industrial and Corporate Change, Vol. 8 (1999), S. 353-408 (3) Vgl. Witt, U.: Individualistische Grundlagen der Evolutorischen Ökonomik. Tübingen 1987, Kap. III und Witt, U.: Learning to Consume - A Theory of Wants and the Growth of Demand. In: Journal of Evolutionary Economics, Vol. 11 (2001), S. 23-36.

(4) Vgl. Witt, U.: Genes, Culture, and Utility. Papers on Economics and Evolution, Nr. 0009 (2000), Max-Planck-Institut Jena. (5) Millenson, J.R.: Principles of Behavior Analysis. New York 1967.

(6) Ruprecht, W.: The Historical Development of the Consumption of Sweeteners - A Learning Approach. Papers on Economics and Evolution, Nr. 0104 (2001), Max-Planck-Institut Jena.

(7) Hirsch, F.: Social Limits to Growth. Cambridge MA 1978.

(8) Scitousky, T.: The Joyless Economy: An Inquiry Into Human Satisfaction and Consumer Dissatisfaction. Oxford 1976. (9) Siehe Lane, R.E.: The Loss of Happiness in Market Economies. New Haven 2000.

\section{Der Autor}

Prof. Dr. Ulrich Witt ist Direktor der Abteilung Evolutionsökonomik am Max-Planck-Institut zur Erforschung von Wirtschaftssystemen (MPIEW).

Kontakt: MPIEW, Kahlaische Str. 10, D-07745 Jena, Tel. 03641/ 686-801, Fax -868,

E-Mail: witt@mpiew-jena.mpg.de 
(c) 20I0 Authors; licensee IÖW and oekom verlag. This is an article distributed under the terms of the Creative Commons Attribution Non-Commercial No Derivates License (http://creativecommons.org/licenses/by-nc-nd/3.o/), which permits unrestricted use, distribution, and reproduction in any medium, provided the original work is properly cited. 